\title{
Femoral Test Bed for Impedance Controlled Surgical Instrumentation
}

\author{
Christian Brendle ${ }^{1}$, Annegret Niesche², Alexander Korff², Klaus Radermacher², Steffen \\ Leonhardt ${ }^{1}$ \\ ${ }^{1}$ Philips Chair for Medical Information Technology, Helmholtz Institute for Biomedical Engineering, RWTH Aachen \\ University, Pauwelsstrasse 20, 52074 Aachen, Germany \\ ${ }^{2}$ Chair for Medical Engineering, Helmholtz Institute for Biomedical Engineering, RWTH Aachen University, Pauwelsstr. \\ 20, 52074 Aachen, Germany
}

Corresponding author: brendle@hia.rwth-aachen.de

\begin{abstract}
The risk for patients during the standard procedure of revision of cemented artificial hip joints is unsatisfactorily high due to its high level of invasiveness and limited access to the operative field. To reduce this risk we are developing an Impedance Controlled Surgical Instrumentation (ICOS) system, which aims to establish real-time control during a Bone Cement (BC) milling process. For this, the relationship between the thickness of the BC and its frequency-dependent electrical impedance is used to estimate the residual $\mathrm{BC}$ thickness. The aim is to avoid unintended cutting of bone by detecting the passage of the $\mathrm{BC} /$ bone boundary layer by the milling head. In a second step, an estimation of the residual $\mathrm{BC}$ thickness will be used to improve process control. As a first step towards demonstrating the feasibility of our approach, presented here are experimental studies to characterize the BC permittivity and to describe the process in detail. The results show that the permittivity properties of BC are dominated by its polymethyl methacrylate (PMMA) fraction. Thus, PMMA can be used as a substitute for future experiments. Furthermore, a Femoral Test Bed (FTB) was designed. Using this setup we show it is feasible to accurately distinguish between slightly different thicknesses of BC.
\end{abstract}

Keywords: Bioimpedance spectroscopy, surgical instrumentation, artificial hip replacement, Bone Cement milling.

\section{Introduction}

In industrialized countries procedures such as total hip replacement are frequently performed to improve quality of life and maintain mobility of the aging society. For example, in Germany in $2008 \geq 150000$ primary total hip replacements were implanted and the number of cases is increasing [2]. The major part of the implanted artificial hip joint is cemented $[6,10]$; however, a systematic review showed that only 88 $95 \%$ of the cemented artificial hips are revision-free after 10 years [4]. This implies that hip revision surgery has now become a standard procedure. For example, in Germany alone, in $2008, \geq 22500$ revisions of total hip arthroplasties took place [3].

During revision surgery, the old BC has to be removed to ensure fixation of the new implant [9]. Generally this is done by manual control of surgical hammer and chisel. However, due to restricted access to the operative field, lateral femur windowing may be used to help resolve this problem. Unfortunately, these standard procedures are highly invasive and the risk of unintended damage to the bone or vessels is very high [14].

An alternative approach is to use milling tools for removal of $\mathrm{BC}$. To acquire information on the spatial characteristics of $\mathrm{BC}$, which is necessary for tool navigation, ultrasound or computed tomography (CT)-

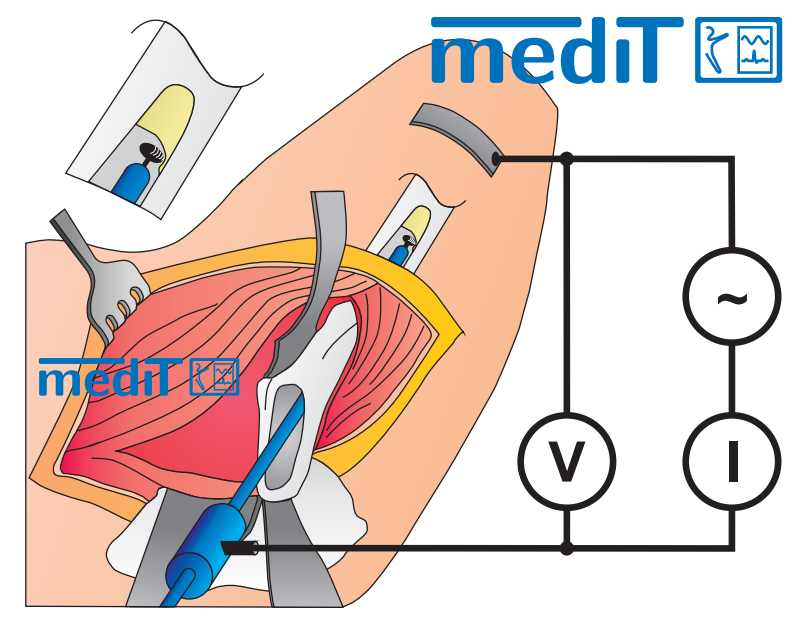

Figure 1: Impedance Controlled Surgical Instrumentation: planned operative scenario.

based measurements are used. This requires an additional step before or during the operation, apart from the actual milling process $[6,5,8]$. However these additional steps cause stress, and CT imaging exposes the patient to ionizing radiation.

To avoid these disadvantages, our approach uses real-time control of the milling process during $\mathrm{BC}$ removal. Figure 1 presents the planned surgical scenario. Through the milling head, an alternating cur- 
rent is applied to the patient and the voltage between the milling head in the femur and the patient's leg skin is measured. The measured values are used to calculate the spectral two-point bioimpedance from which we want to deduce the thickness of the residual $\mathrm{BC}$ in the femur.

\section{Permittivity characterization of Bone Cement}

BC consists mainly of PMMA. The ICOS system utilizes the high electrical impedance of PMMA, which is several orders of magnitude higher than the impedance of human tissue. To illustrate this, we compare the impedance fractions of the different layers of tissue between the femur center and the skin surface of the leg using a two-point Bioimpedance Spectroscopy (BIS) measurement. Body tissue with the highest impedance fraction is dry skin, despite its relatively small thickness. Comparison of the absolute impedance values of two commensurate probes out of PMMA and dry skin shows that the PMMA value of $110 \mathrm{kHz}$ is 400 times higher than the value of dry skin $[11,1]$. Thus, the BC impedance presents the major part of the measured impedance in the ICOS system.

To verify the characteristic $\mathrm{BC}$ permittivity and the relation between its thickness and electric impedance we analyzed probes from Biomet $\AA$ Bone Cement $\mathrm{R}$ using a capacitor measurement setup. The $\mathrm{BC}$ capacitor impedance was measured with an Agilent E4890A LCR Meter in the range of $2 \mathrm{kHz}$ to $2 \mathrm{MHz}$. To calculate characteristic BC permittivity values, from the measured values we used eq. (1) (with sample dimensions: side length $a=10 \mathrm{~mm}$ and thickness $d=1,2$, and $3 \mathrm{~mm}$ ).

$$
\begin{aligned}
\underline{\mathbf{Y}}_{B C} & =G+j \cdot B \\
& =\frac{a^{2}}{d} \cdot j \cdot \omega \cdot \varepsilon_{0} \cdot\left(\varepsilon_{r}^{\prime}-j \cdot \varepsilon_{r}^{\prime \prime}\right) \\
\Longrightarrow \varepsilon_{r}^{\prime} & =\frac{B \cdot d}{a^{2} \cdot \omega \cdot \varepsilon_{0}} \\
\varepsilon_{r}^{\prime \prime} & =\frac{G \cdot d}{a^{2} \cdot \omega \cdot \varepsilon_{0}}
\end{aligned}
$$

Figure 2 presents the characteristic BC permittivity. The red curves represent the mean values of 36 independent measurement series and the grey area indicates the standard deviation for each frequency. $\varepsilon_{r}^{\prime}$ represents the stored energy and the $\varepsilon_{r}^{\prime \prime}$ characterizes the loss of energy within the medium. The diagram confirms that BC dielectric properties are characterized by the PMMA's properties. In the complex $\varepsilon_{r^{-}}$ plane the curve of the measured BC (red) is shifted about $10 \%$ to the right relative to the values reported in the literature for PMMA (black) [11]. This $10 \%$ shift can also be observed in the frequency dependent
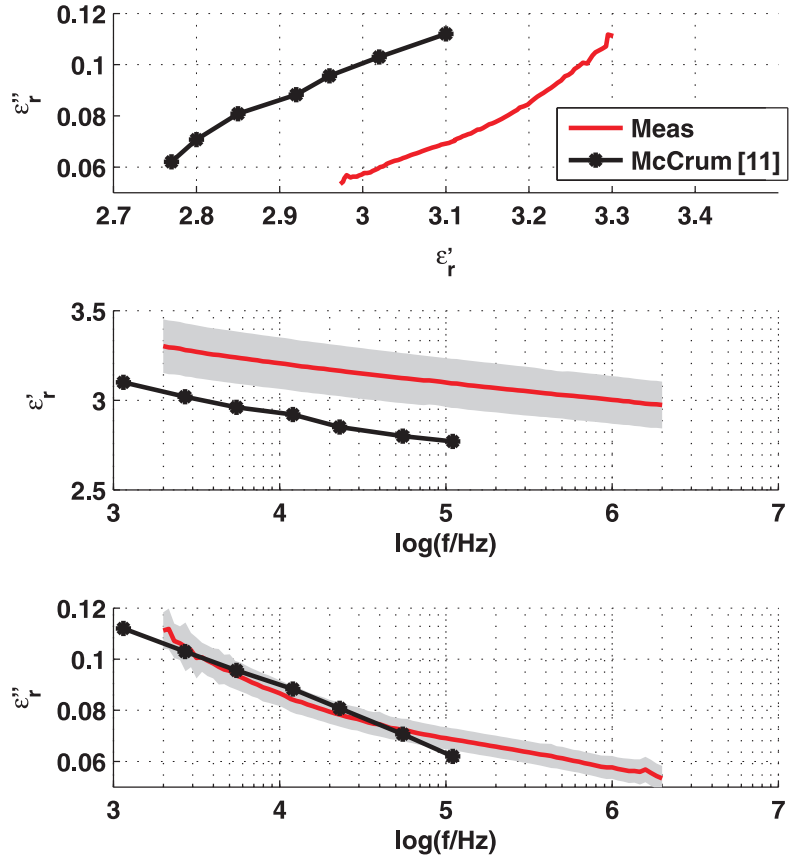

Figure 2: Permittivity measurements of BC (red) with standard deviation (grey) according to McCrum et al. permittivity values for PMMA (black) [11]: Complex permittivity plane (top), real part of the permittivity $\varepsilon_{r}^{\prime}$ over frequency $f$ (middle), imaginary part of the permittivity $\varepsilon_{r}^{\prime \prime}$ over frequency $f$ (bottom).

illustration of $\varepsilon_{r}^{\prime}$ in Figure 2 (middle band). The $\varepsilon_{r}^{\prime \prime}$ of $\mathrm{BC}$ values are in accordance with the published values for PMMA.

Based on these measurements, it is acceptable to use PMMA dummies for our experiments to demonstrate the functionality of the ICOS system and to describe the actual process.

\section{Design of Femoral Test Bed}

The FTB presented in Figure 3 is used as a representation of the scenario for the adult human leg. Thus, the geometry of the FTB is defined as follows. The basis is a cylindrical bowl with an inner diameter of $15 \mathrm{~cm}$ as an approximation of thigh diameter. The relevant volume of the impedance measurement is determined by the path of the electrical current. For this reason, the inner height of the bowl is set at $4 \mathrm{~cm}$. The anatomical femur position is $3.25 \mathrm{~cm}$ off-center. However, for the first measurements the center position is required for geometric invariance. Therefore, a carrier for the PMMA dummy (Figure 4, green) can be mounted in the middle of the bowl and $3.25 \mathrm{~cm}$ off-center. The dummy, representing the BC, is a hollow cylinder with varying wall thicknesses (1, $1.5,2$, and $2.5 \mathrm{~mm}$ ) in the upper part. In addition 


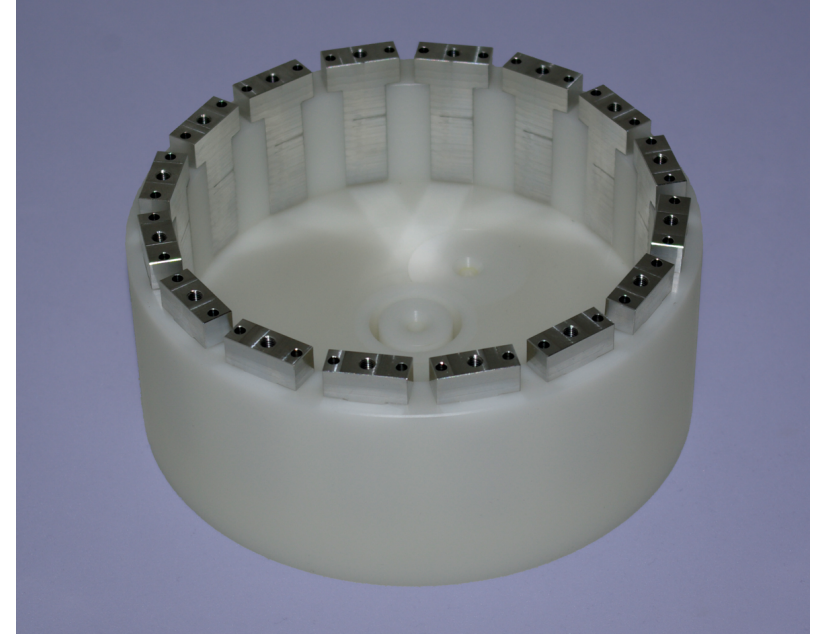

Figure 3: FTB with 16 T-shaped electrodes of the inner wall.

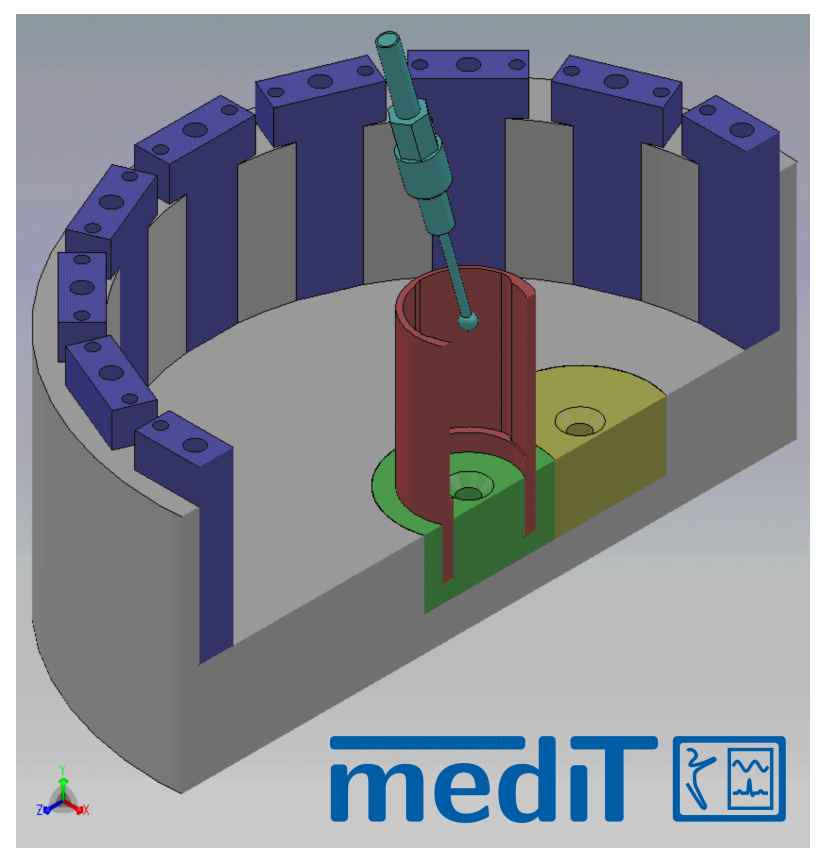

Figure 4: Cross-sectional view of the measurement setup: bowl (grey), T-shaped electrodes (blue), carrier (green), dummy plate (yellow), PMMA dummy (red) and point electrode (cyan).

a dummy plate (Figure 4, yellow) for the free carrier position is provided.

The space between the dummy and bowl wall is filled with a conductive material as a substitute for human tissue. For these experiments we use $\mathrm{NaCl}$ solutions with an adjusted conductivity. For future experiments agar-agar gel compositions will be used [13]. These gels acquire a solid structure after a certain curing time. During the casting process of agar-agar gel, its temperature can rise to $80^{\circ} \mathrm{C}$. This has to be considered in the choice of bowl material. Moreover, the bowl needs to be a good isola-

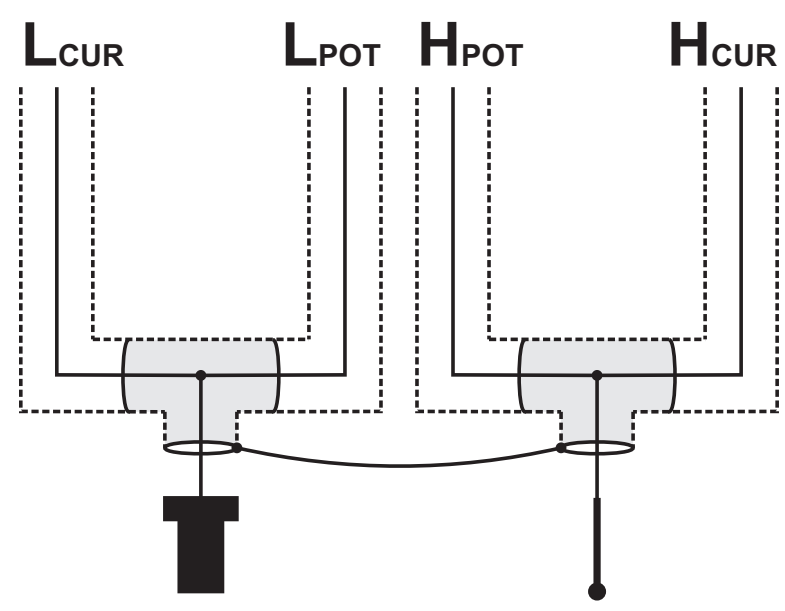

Figure 5: Wiring diagram for the first BIS measurement: the two-terminal method.

tor to avoid unwanted effects on the measurements. Therefore, polyoxymethylene (POM) is a good choice for the bowl material due to its negligible electrical conductivity and its thermal stability in the required temperature range [7]. Furthermore, the T-shaped electrodes in the wall should be replaceable for updates in the experimental setting. In addition, we need 16 uniformly distributed electrodes to be able to use this FTB in Electrical Impedance Tomography (EIT) experiments.

The first measurement setup will be BIS measurements between the point electrode (Figure 4, cyan) in the middle, and one T-shaped electrode (Figure 4, blue). The bowl will be filled with $\mathrm{NaCl}$ solution with a conductivity of $850 \mu \mathrm{S} / \mathrm{cm}$, similar to the conductivity of cancellous bone [1].

\subsection{Electrical contacting}

The Agilent E4890A LCR Meter has four BNC connectors used for impedance measurements, two for the low connector pair and two for the high connector pair. In each case, one of these two BNC connectors is used to apply the measurement current and the other for the electrical potential measurement. The LCR Meter is connected to the FTB using a twoterminal measurement configuration (Figure 5) with four $0.5 \mathrm{~m}$ coaxial cables. The current and the potential cable to the FTB electrodes are connected using BNC T-Connectors (Figure 5, grey). The cladding contacts of the two BNC T-Connectors are connected to limit the effects of measurement incidents.

The measurement range is set between $200 \mathrm{kHz}$ and $2 \mathrm{MHz}$. The LCR Meter low connector pair is connected to one T-shaped electrode (Figure 4, blue) and the high connector pair is connected to the point electrode (Figure 4, cyan). This is realized using screwed solder terminal lugs, which are connected to the inner 


\begin{tabular}{ccc}
\hline Two-sample t-test & p-value & $\begin{array}{c}\text { Significant } \\
\text { difference }\end{array}$ \\
\hline$\left|Z_{1,0 \mathrm{~mm}}\right|<\left|Z_{1,5 \mathrm{~mm}}\right|$ & $0.38 \%$ & yes \\
\hline$\left|Z_{1,5 \mathrm{~mm}}\right|<\left|Z_{2,0 \mathrm{~mm}}\right|$ & $1.21 \%$ & yes \\
\hline$\left|Z_{2,0 \mathrm{~mm}}\right|<\left|Z_{2,5 \mathrm{~mm}}\right|$ & $5.06 \%$ & no \\
\hline
\end{tabular}

Table 1: Two-sample t-test with significance level $\alpha=5 \%$.

conductor of the two BNC T-connectors (Figure 5, grey).

\section{First results}

The impedance curves in the $Z$-plane (Figure 6, top) show the capacitive characteristic of the PMMA dummy wall. Assuming a similar scale for both diagram axes, the curves are almost parallel to the negative $y$-axis and aspire to the origin of the $Z$-plane with increasing frequency. This characteristic is confirmed by the decrease of the absolute value of the mean impedance value from four measurement series over the frequency (Figure 6, bottom left). This plot also shows that our measurement range extends to $\mathrm{M} \Omega$ values. Figure 6 (bottom right) presents the mean impedance with standard deviation at $2 \mathrm{MHz}$ as a function of the wall thickness. This plot shows that the probability distributions of the measurement series for different thicknesses intersect within their range of the single standard deviation for greater wall thicknesses. With smaller wall thicknesses the relative standard deviation decreases from $8.31 \%$ at $2.5 \mathrm{~mm}$ to $4.34 \%$ at $1 \mathrm{~mm}$.

To characterize the sensitivity of the first experiments, a two-sample t-test was performed with the absolute impedance values at $2 \mathrm{MHz}$. For this we used a left-tail test taking into account that the mean impedance of smaller wall thickness is lower [12]. In addition, we assume unequal variances. The results of the t-test (Table 1) show that the measured difference of the impedance values is significant for smaller wall thicknesses. Due to the higher standard deviations for greater wall thicknesses it is not possible to show significant differences.

\section{Conclusion}

The FTB represents the surgical operative setting. It allows repeatable arrangements of experimental settings with PMMA dummies as a substitute for the BC. For medical application, the sensitivity of the ICOS system at smaller wall thicknesses $(<2 \mathrm{~mm})$ is important because the risk of damage to patient tissue is increased in this range. The results of the first
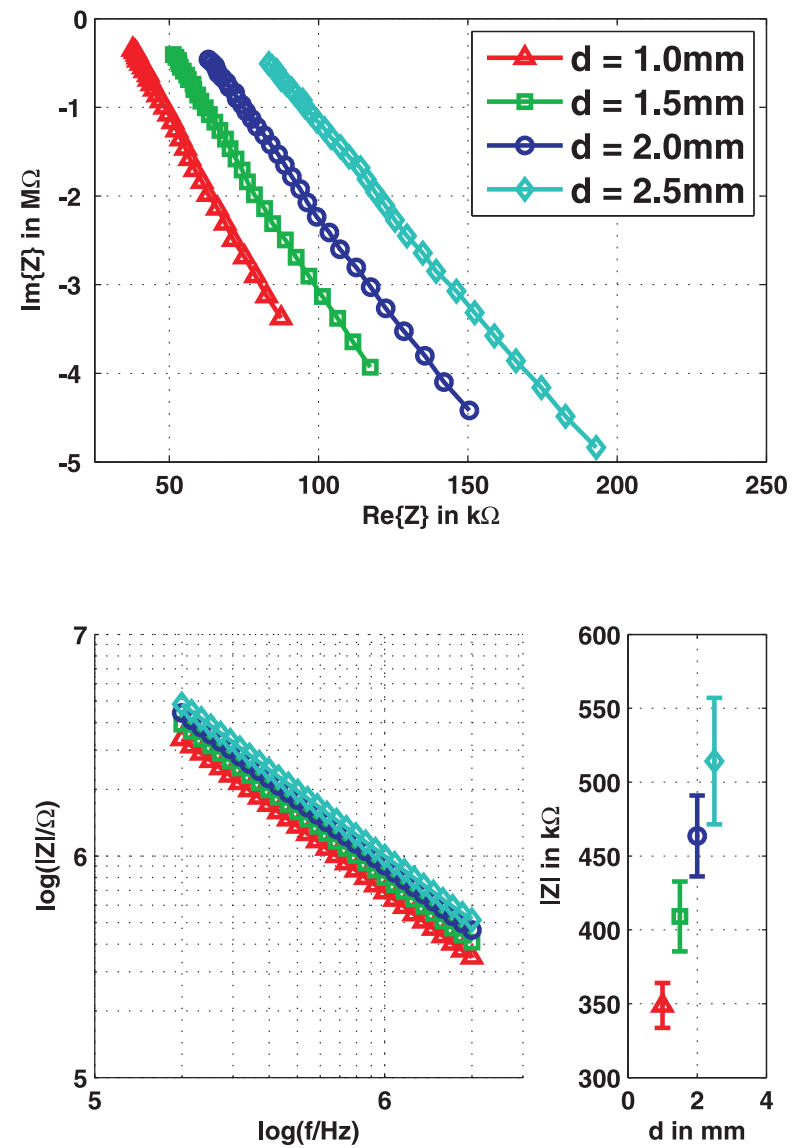

Figure 6: Impedance measurements in the FTB with a hollow cylindrical PMMA dummy with different wall thicknesses $d$. Top: $Z$-plane with mean impedance curves. Bottom left: Absolute value of the mean impedance as function of the frequency. Bottom right: Absolute impedance mean values with standard deviation at $2 \mathrm{MHz}$ as a function of wall thickness.

experiments with a non-rotating point electrode indicate the suitability of the measurement system for this purpose. For more valid values, a larger sample size is required.

In the future, additional experiments will support further development of the ICOS system. For example, a theoretical model of the electrical conduction in the setup is needed which also takes into account the effects of surface conduction. In addition, the influence of current transmission on the rotating milling shaft and the transmission from the rotating milling head to the BC needs to be characterized.

\section{Acknowledgements}

The authors thank the Federal Ministry of Education and Research for financial support of the research project Impedance Controlled Surgical Instrumentation (07EZ1005). 


\section{References}

[1] R. Andreuccetti, D.and Fossi, C. Petrucci. Dielectric properties of body tissues: in the frequency range $10 \mathrm{~Hz}-100 \mathrm{GHz}, 1997-2007$. http: //niremf.ifac.cnr.it/tissprop/, [2012-09$10]$.

[2] O. Boy, S. Hahn, E. Kociemba. Hüft Endoprothesen Erstimplantation. In BQSQualitätsreport, pp. 146-153. 2008.

[3] O. Boy, S. Hahn, E. Kociemba. Hüft-Endoprothesenwechsel und -komponentenwechsel. In BQS-Qualitätsreport, pp. 154-160. 2008.

[4] Kelly L. Corbett, Elena Losina, Akosua A. Nti, et al. Population-based rates of revision of primary total hip arthroplasty: A systematic review. PLoS ONE 5(10):e13520, 2010.

[5] R.E. Ellis. From scans to sutures: Computerassisted orthopedic surgery in the twenty-first century. In Engineering in Medicine and Biology Society, 2005. IEEE-EMBS 2005. 27th Annual International Conference of the, pp. 7234-7237. 2005.

[6] M. de la Fuente, J.A.K. Ohnsorge, E. Schkommodau, et al. Fluoroscopy-based 3-d reconstruction of femoral bone cement: a new approach for revision total hip replacement. Biomedical Engineering, IEEE Transactions on 52(4):664-675, 2005 .

[7] Jurima Dichtungen GmbH. POM - C Datenblatt, 2012. http://www.jurima-gmbh.de/ downloads/pomcdatenblatt.pdf, [2012-09-10].
[8] S. Heger, T. Vollborn, M. Niggemeyer, et al. Robot integrated ultrasound geometry-scanning for trackerless bone cement detection in rthr. In Ultrasonics Symposium (IUS), 2010 IEEE, pp. 845-848. 2010.

[9] P. L. S. Li, P. J. Ingle, J. K. Dowell. Cementwithin-cement revision hip arthroplasty; should it be done? Journal of Bone 6 Joint Surgery, British Volume 78-B(5):809-811, 1996.

[10] Henrik Malchau, Peter Herberts, Peter Söderman, Anders Odén. Prognosis of total hip replacement: Update and validation of results from the swedish national hip arthroplasty registry 1979-1998. In 6rth Annual Meeting of the American Academy of Orthopaedic Surgeons, Orlando, USA, pp. 1-16. 2000.

[11] N. G. McCrum, B. E. Read, G. Williams. Anelastic and Dielectric Effects in Polymeric Solids. John Wiley \& Sons Ltd, 1967.

[12] MathWorks@. Matlab product documentation: Two-sample t-test, 2012. http://www.mathworks.de/help/toolbox/ stats/ttest2.html, [2012-09-10].

[13] Tong In Oh, Hwan Koo, Kyung Heon Lee, et al. Validation of a multi-frequency electrical impedance tomography (mfEIT) system KHU Mark1: impedance spectroscopy and timedifference imaging. Physiological Measurement 29(3):295, 2008.

[14] J. Pfeil. Hüftchirurgie. 1st ed., Steinkopff, 2008. 